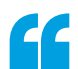

\section{After 3 years of hard work we succeeded in making samples in which most of the exfoliated species are ribbons}

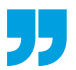

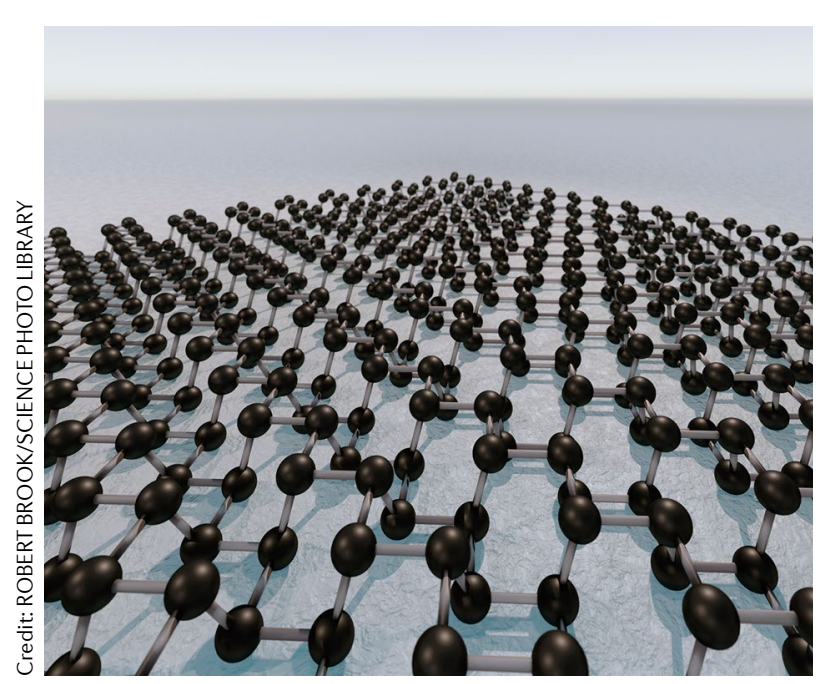

\title{
Cutting phosphorene nanoribbons
}

Shaping 2D materials as nanoribbons can unlock new phenomena and applications, but is no easy feat. In particular, a method to produce phosphorene nanoribbons (PNRs) has eluded researchers for years. Now, Mitch Watts, Chris Howard and colleagues report in Nature a scalable process to produce high-quality PNRs.

Phosphorene is a $2 \mathrm{D}$ material with high carrier mobility, high optical and UV absorption and a bandgap that depends on the number of layers in the sample; moreover, the presence of two different $\mathrm{P}-\mathrm{P}$ bond lengths makes the properties of phosphorene unusually anisotropic. Owing to these characteristics, phosphorene has attracted substantial attention in recent years, but theory predicts even more exciting properties for PNRs, including the emergence of exotic phenomena, such as topological and excitonic effects, that could be harnessed in devices.

However, producing PNRs is far from trivial. When it comes to nanoribbon production, top-down approaches normally fail as they cannot deliver the desired uniform widths and crystallinity. On-surface synthesis based on the polymerization of molecular precursors has proved very successful for the synthesis of graphene nanoribbons, but cannot be extended to phosphorene because of the current lack of appropriate precursors.

The authors used a two-step process in which crystals of bulk phosphorus were first intercalated with lithium ions and then immersed in a solvent and agitated. "Actually we didn't set out to make ribbons at all!" comments Watts. "We were trying to make $2 \mathrm{D}$ phosphorene by applying our recently reported method for forming 2D materials in liquids." In this method, the insertion of alkali metal ions between the sheets of a layered material negatively charges the layers. The resulting 'layered material salt' is then placed in an appropriate solvent, in which the negatively charged sheets dissolve to form solutions of monolayers. "We attempted this method with black phosphorus without success, but after several different attempts and modifications we noticed some ribbon-like fragments," continues Watts. "After 3 years of hard work we succeeded in making samples in which most of the exfoliated species are ribbons."

Alkali metal ions intercalated in black phosphorus diffuse rapidly along the zigzag crystallographic direction, forming stripes. The combination of mechanical strain and electron doping can break the longer P-P bonds, resulting in cracks in the zigzag direction that make black phosphorus flakes split; this is why ion intercalation in black phosphorus results in the formation of nanoribbons rather than 2D sheets.

The nanoribbons, which can be several tens of micrometres long and are very stable under an inert atmosphere but oxidize when exposed to air, similar to phosphorene, were then characterized by transmission electron microscopy and atomic force microscopy (AFM), revealing they are mostly monolayer and remarkably flexible, that their height and width is very uniform along their length and that their long axis is always aligned in the zigzag direction. "Team members and collaborators at Bristol have built an incredibly fast AFM, which meant we could take images at video rate speeds," explains Howard. "This high-speed AFM allowed us to investigate the ribbons' morphology in far greater detail than is typical for nanomaterials characterization."

The scalability of this production method will enable not only the investigation of the exotic properties predicted for PNRs, but also their study for incorporation in practical devices, such as thermoelectric devices, fast-charging batteries, efficient solar cells and high-speed integrated electronic circuits. "We'd like to see many experiments on PNRs: we provided substantial detail in the methods section of the paper and hope that many other groups will start making PNRs and exploring their properties," concludes Howard. "Meanwhile, we will continue with experiments on their properties, following up some very interesting preliminary work on surface plasmons, and with our collaborators we plan to immediately test the ribbons for battery applications."

Giulia Pacchioni

ORIGINAL ARTICLE Watts, M. C. et al.

Production of phosphorene nanoribbons. Nature 568, 216-220 (2019)

FURTHER READING Cullen, P. L. et al. lonic solutions of two-dimensional materials. Nat. Chem. 9, 244-249 (2017) 